\title{
Asignación Óptima de Presupuesto para Mejoramiento de la Calidad del Servicio en Sistemas de Distribución usando Algoritmo Genético No-Dominado II (NSGA-II) y un Algoritmo Memético
}

\author{
Luis F. Rojas-Ríos ${ }^{(1)}$, Jesús M. López-Lezama ${ }^{(2)}$ y Nicolás Muñoz-Galeano(2) \\ (1) Empresas Públicas de Medellín (EPM), Cr 58 No 42-125, Medellín - Colombia \\ (e-mail: luis.rojas@epm.com.co) \\ (2) Grupo de Investigación GIMEL, Departamento de Ingeniería Eléctrica, Facultad de Ingeniería, \\ Universidad de Antioquia, Calle 70 No. 52-21, Medellín - Colombia (e-mail: jmaria.lopez@udea.edu.co; \\ nicolas.munoz@udea.edu.co)
}

Recibido Ago. 3, 2015; Aceptado Oct. 5, 2015; Versión final Nov.4, 2015, Publicado Feb. 2016

\begin{abstract}
Resumen
Se presenta un modelo de asignación óptima de presupuesto para mejoramiento de calidad del servicio en sistemas de distribución. El modelo consiste en un problema multiobjetivo que busca al mismo tiempo minimizar el costo de mantenimiento en sistemas de distribución y maximizar la reducción de la tasa de fallas. Este último objetivo se evalúa a través del indicador SAIFI (Frecuencia Media de Interrupción del Sistema). Para resolver el modelo propuesto se implementaron dos algoritmos poblacionales: Algoritmo Genético No-Dominado II (NSGA-II) y un Algoritmo Memético. Se realizan pruebas con dos sistemas eléctricos reales del Departamento de Antioquia en Colombia de 100 y 200 nodos, mostrando la aplicabilidad del modelo propuesto. Los frentes de Pareto óptimos obtenidos en la solución del problema muestran un set de posibles soluciones que representan un compromiso entre ambos objetivos y le dan al operador de red un estimado de cuánto debe invertir en mantenimiento para lograr un valor deseado del indicador SAIFI.
\end{abstract}

\section{Optimal Budget Assignment for Service Quality Improvement in Distribution Systems using Non-Dominated Sorting Genetic Algorithm II (NSGAIl) and Memetic Algorithm}

\begin{abstract}
This paper presents an optimal budget assignment model for improving quality service in distribution systems. The model consists on a multi objective problem which aims at minimizing maintenance costs in distribution systems while maximizing the reduction of faults rate. This last objective is measured through the SAIFI indicator (System Average Interruption Frequency Index). To solve the proposed model two algorithms were implemented: NSGAll (Non-Dominated Sorting Genetic Algorithm II) and a Memetic Algorithm. Several tests were performed with two real electrical systems of 100 and 200 nodes in the Department of Antioquia in Colombia, showing the applicability of the proposed approach. The optimal Pareto fronts obtained in the problem solution show a set of available options that represent a trade-off between both objectives and provides the system operator with an estimate of how much to invest in maintenance to achieve a desired value of the SAIFI indicator.
\end{abstract}




\section{INTRODUCCIÓN}

En el negocio de la distribución de energía eléctrica los organismos reguladores y de control definen indicadores de calidad para la continuidad en la prestación del servicio que deben ser atendidos por las empresas de distribución. Estos indicadores están relacionados con la duración y frecuencia de las interrupciones a los usuarios. El no cumplimiento de ciertos niveles en estos indicadores puede llevar a las empresas distribuidoras a pagar multas o indemnizaciones a los usuarios afectados. Dada la complejidad de los sistemas de distribución y la variabilidad del desempeño de la confiabilidad en estos sistemas se hace necesaria la aplicación de herramientas de optimización que faciliten la toma de decisiones a los ingenieros de planeación. En la literatura especializada se han propuesto diferentes metodologías para programar el mantenimiento de las redes de distribución con el objetivo de impactar en los índices de calidad y continuidad del servicio.

En (Xianchao et al., 2008) se presenta una metodología para programar de acciones de mantenimiento en sistemas de distribución mediante un algoritmo genétio. El algoritmo propuesto incluye un operador de mutación basado en una búsqueda tabú para mentener la diversidad de la poblacion y evitar la convergencia prematura del algoritmo. Los autores proponen minimizar el tiempo de mantenimiento pero no tienen en cuenta variables asociadas al costo del mismo. En (Abiri-Jahromi et al., 2009) se presenta un modelo de programación lineal entera mixta para programar el mantenimiento de largo plazo en sistemas de distribución. El objetivo es identificar el mejor escenario de mantenimiento en términos de costo/eficiencia teniendo en cuenta restricciones de confiabilidad y un presupuesto asignado. La formulación propuesta por los autores utiliza factores de riesgo desacoplados y un modelo de transición de estado basado en un árbol de decisión. Para cada línea del sistema se utilizan tres variables binarias asociadas con actividades específicas de mantenimiento. En este caso los indices de calidad se proponen como restricciones del modelo y no como parte del objetivo del problema de optimización. El modelo propuesto es probado en sistemas de 11 y 110 alimentadores.

En (Teera-archariyakul, et al, 2010) se propone un método para la asignación del presupuesto para mejorar indicadores de calidad y además la forma de fijar metas de mejoramiento cuantitativas de indicadores de calidad. Con base en la técnica de Mantenimiento Basado en Confiabilidad se identifican las fallas candidatas a ser gestionadas con el objetivo de disminuir la tasa de ocurrencia. Además, mediante encuestas a expertos se determina el impacto en la confiabilidad. Luego se formula la asignación óptima del presupuesto de mantenimiento por región para una empresa de distribución. El costo y el impacto en la confiabilidad se emplean para priorizar las acciones de mantenimiento resolviendo un problema de asignación de recursos fácilmente aplicable a problemas de baja complejidad. En (Certa et al., 2011) se presenta una metodología para obtener el frente de Pareto óptimo en un problema de mantenimiento multiobjetivo que pretende minimizar el costo de mantenimiento total y el tiempo del mismo. La metodología se basa en algoritmos evolutivos y se presenta una aplicación en el mantenimiento de una unidad naval militar. En (Yongmei et al., 2011) se prone un algoritmo genético para reconfiguracion de redes y programación de mantenmientos en sistemas de distribución. Para reducir el espacio de búsqueda se adoptan operadores que permiten generar soluciones factibles en la codificación del algoritmo. La función objetivo consiste en la minimización de pérdidas activas y el costo de mantenimiento. Se presentan resultados en el sistema de prueba IEEE de 33 barras. En (Arias, et al., 2012) los autores proponen una programación óptima del mantenimiento de redes de distribución con el objetivo de minimizar la energía no servida debido a fallos ocasionados por vegetación. La solución del modelo propuesto se logra mediante un algoritmo genético especializado. El problema es abordado desde una perspectiva mono-objetivo considerando como restricción los recursos disponibles para eferctuar mantenimiento en funcion de los metros de red donde se pueda hacer poda de la vegetacion. El modelo es probado en un sistema de 19 alimentadores para un horizonte de tiempo de un año dividido en 4 periodos. En (Arya y Choube, 2013) se proponen herramientas para la programación óptima de las actividades de mantenimiento que tienden a la optimización de costos y, de forma indirecta, al mejoramiento de los indicadores de calidad.

En la literatura técnica se encuentra de forma recurrente el uso de metaheurísticas para asignación de presupuesto y programación de mantenimiento en redes de distribución. En este artículo se pretende aportar a la discusión al respecto mediante la implementación y comparación de dos técnicas multiobjetivo: NSGA-II (Non-dominated Sorting Genetic Algorithm) y un Algoritmo Memético. El funcionamiento del algoritmo NSGAll se basa en una clasificación no dominada de los individuos en cada generación en la cual se organiza la poblacion en diferenes frentes de Pareto. En cada ciclo los nuevos individuos se generan a partir de las configuraciones de los frentes no dominados (Mazidi et al., 2013). Este algoritmo ha mostrado ser efectivo en diferenes aplicaciones en ingeniería como se evidencia en (Bahri et al., 2014) y (Hajebrahimi et al., 2014). Por otro lado, los Algoritmos Meméticos que combinan los algoritmos genéticos con operadores de búsqueda local también han mostrado ser efectivos en aplicaciones multiobjetivo (Sun et al., 2014). Existen otras técncias de optimización multiobjetivo como SPEAll (Strength Pareto Evolutionary 
Algorithm II), MOGA (Multi Objective Genetic Algorithm), PAES (Pareto Archived Evolution Strategy), PESAll (Pareto Envelope-based Selection Algorithm II), NPGA (Niched Pareto Genetic Algorithm), MOPSO (MultiObjective Particle Swarm Optimization), etc. Un estudio comparativo de algunas de estas técncias puede ser consultado en (Ghoreishi et al., 2015). Para efectos de este artículo se han selecciondo los algorimos NSGAll y un Algoritmo Memético por ser dos de los más representativos y comunmente utilizados en aplicaciones similares al problema bajo estudio.

Los objetivos implementados consisten en la minimización del costo de mantenimiento y la maximización en la reducción de la tasa de fallas. El trabajo presentado en este artículo se diferencia de otros reportados en la literatura técncia en los siguientes aspectos: i) el índice de calidad se propone como un de los objetivos del problema y no como una restriccción; ii) los algoritmos descritos son evaluados con sistemas reales de 100 y 200 nodos y iii) Se aborda el problema desde una perspectiva multi-objetivo maximizando la calidad del servicio y minimizando el costo de la misma. La principal ventaja de los algoritmos propuestos radica en el hecho de obtener un set de soluciones sobre las cuales el operador de red puede seleccionar la más apropiada en términos de su relación beneficio/costo.

\section{FORMULACION DEL PROBLEMA}

Como se mencionó anteriormente, el modelo propuesto involucra dos funciones objetivo: i) la minimización del costo de mantenimiento de la red de distribución y ii) la maximización de la reducción de la tasa de fallas. La reducción de la tasa de fallas se evalúa a través del indicador SAIFI (Frecuencia Media de Interrupción del Sistema) que indica el número promedio de veces que un cliente del sistema sufre una interrupción durante el periodo de tiempo analizado, como se indica en la ecuación (1). Por otro lado, el costo de mantenimiento está dado por la ecuación (2).

$\operatorname{Max} \Delta$ SAIFI $=\frac{1}{\mathrm{Ct}} \sum_{\mathrm{i} \in \text { Nodos }} \lambda_{\mathrm{i}} * \mathrm{c}_{\mathrm{i}} \prod_{\mathrm{K}=\mathrm{i}+1}^{\mathrm{n}} \mathrm{x}_{\mathrm{i}}$

Donde $x_{i}$ es la variable binaria que define si el tramo de red es intervendio con acciones de mantenimiento, $C_{i}$ y $C_{t}$ son parámetros que indican la cantidad de clienes afectados por una interrupcion del servio y el número total de clienes del sistema eléctrico, respectivamente. Finalmente $\lambda_{\mathrm{i}}$ es el parámetro que indica el número de interrupciones del elemento por año.

Min costo $=\sum_{i \in \text { Nodos }} d_{i} * l_{i} \prod_{K=i+1}^{n} x_{i}$

En este caso $x_{i}$ es la variable binaria que define si el tramo de red es intervendio con acciones de mantenimiento, mientras $d_{i}$ y $l_{i}$ son parámetros que indican el costo de intervenir un kilómetro de red y los kilómetros de red aguas abajo de los elementos de seccionamiento, respectivamente.

Inicialmente se considera un problema sin restricciones donde se pretende conocer el frente de Pareto de la solución, con el fin de identificar el potencial de mejora del SAIFI y el costo necesario. Una vez se defina una meta de costo $k$, o mejora del SAIFI $e$, se pueden incluir las restricciones dadas por (3) y (4).

$$
\begin{aligned}
& \frac{1}{\mathrm{Ct}} \sum_{\mathrm{i} \in \text { Nodos }} \lambda_{\mathrm{i}} * \mathrm{c}_{\mathrm{i}} \prod_{\mathrm{K}=\mathrm{i}+1}^{\mathrm{n}} \mathrm{x}_{\mathrm{i}} \leq \mathrm{e} \\
& \sum_{i \in \text { Nodos }} d_{i} * l_{i} \prod_{K=i+1}^{n} x_{i} \leq k
\end{aligned}
$$

La variable de decisión del problema está dada por la variable binaria $x_{i}=\{0,1\}$; Si esta variable toma el valor 1 , indica que el tramo correspondiente es candidato a ser intervenido o mantenido, aportando a la reducción de la tasa de fallas; si el valor es 0 indica que el tramo no es intervenido.

Una característica particular de los sistemas de distribución de energía eléctrica es que son activos lineales conformados por relativamente pocos componentes que se repiten por cientos de kilómetros. Por esta razón la codificación se hace por punto de corte, seccionamiento o de protección del circuito. De esta forma, cada punto se representa como un nodo a lo largo del circuito. A cada nodo $n$ se le asigna la longitud de red acumulada aguas abajo y los clientes alimentados por éste. Esta información es usada para calcular las funciones objetivo durante la ejecución de los algoritmos de optimización. La calidad del servicio en los circuitos de distribución se evalúa por elemento de seccionamiento, o sea por tramo de red. Es por esto que para cada circuito se estima la tasa de falla de cada tramo de red. En la Tabla 1 se ilustra un ejemplo de la información utilizada para solucionar el problema de optimización. 
Tabla 1: Ejemplo de información para solucionar el problema de optimización

\begin{tabular}{|l|c|c|c|c|c|c|c|c|c|c|}
\hline Nodo & 1 & 2 & 3 & 4 & 5 & 6 & 7 & 8 & 9 & 10 \\
\hline Longitud [km] & 45 & 32 & 2,5 & 3,8 & 1,8 & 1,3 & 0,7 & 7,0 & 1,6 & 3,4 \\
\hline Clientes & 2838 & 338 & 17 & 20 & 25 & 5 & 0 & 61 & 52 & 34 \\
\hline Tasa falla & 4,6 & 1,2 & 1,2 & 1,2 & 1,2 & 3,2 & 1,0 & 3,2 & 3,2 & 1,2 \\
\hline $\begin{array}{l}\text { Costo de Mantenimiento } \\
\text { [Millones COP] }\end{array}$ & 464,4 & 63,0 & 3,7 & 5,6 & 2,6 & 1,9 & 1,1 & 10,3 & 2,3 & 5,0 \\
\hline
\end{tabular}

Los costos de mantenimiento dependen básicamente de la longitud de la red a intervenir. Estos costos contemplan las actividades de inspección de redes, la ejecución de las tareas de mantenimiento y la administración e interventoría de los trabajos. Para estimar los costos de mantenimiento de un kilómetro de red se puede hacer una cuantificación basada en la cantidad de postes intervenidos por día o por la cantidad de postes por kilómetro.

\section{ESTRATEGIA DE SOLUCION}

Para el problema multiobjetivo planteado en la sección anterior se proponen dos estrategias de solución: un Algoritmo Genético denominado NSGAll y un Algoritmo Memético. Ambas técnicas son brevemente descritas a continuación.

\section{Algoritmo Genético de Clasificación No-dominada (NSGA II)}

Los Algoritmos Genéticos pertenecen a las metaheuritsticas poblaciones que pueden ser utilizadas para la solución de problemas mono o multiobjetivo (Narváez et al., 2015), (García et al., 2012). En el caso de problemas multiobjetivo se debe tener en cuenta el concepto de dominancia. Se dice que una solución $x_{1}$ domina a otra solución $x_{2}$ cuando: a) la solución $x_{1}$ no es peor que $x_{2}$ en ninguno de los objetivos y b) la solución $x_{1}$ es estrictamente mejor que $x_{2}$ en al menos un objetivo. Si alguna de estas dos condiciones no se cumple, entonces $x_{1}$ no domina a $x_{2}$. El concepto de dominancia se utiliza para encontrar un conjunto de soluciones no dominadas, denominadas frente de Pareto. EI NSGAll pertenece a la gama de algoritmos evolutivos que permite encontrar este set de soluciones. Dado que ninguna de las soluciones en el conjunto no dominado es absolutamente mejor que las otras, entonces cualquiera de estas constituye una solución aceptable.

La versión inicial de este algoritmo fue desarrollado en 1994, posteriormente, en 2002, se introdujeron mejoras en la eficiencia y diversidad del algoritmo denominándolo NSGAll (Deb and Pratap, 2002). En este algoritmo, a partir de una población de padres Pt (N individuos) se crea una población descendiente Qt $(\mathrm{N}$ individuos). Las dos poblaciones constituyen el conjunto $R t$ de tamaño $2 \mathrm{~N}$. Posteriormente, mediante ordenamiento no dominado se clasifica la población $R t$ en diferentes frentes de Pareto. La nueva población es generada a partir de configuraciones de frentes no dominados. La población empieza a ser construida con el mejor frente no dominado $\left(F_{1}\right)$, continuando con las soluciones del segundo frente $\left(F_{2}\right)$ y así sucesivamente. Como Rt es de tamaño $2 \mathrm{~N}$ y solo se necesitan $\mathrm{N}$ individuos para la población descendiente se deben descartar $\mathrm{N}$ configuraciones. Se prefieren los individuos que pertenezcan al menor frente, si ambos pertenecen al mismo frente se prefiere la solución con menos soluciones alrededor. La idea es que se promuevan las configuraciones que aseguren diversidad dentro del mismo frente de Pareto. Cuando la población en su totalidad converge al frente de Pareto óptimo, el algoritmo asegura que las soluciones están distanciadas unas de las otras. En la Fig.1 se ilustra el proceso de selección de individuos del NSGAll. Una descripción detallada del NSGAll puede ser consultada en (Deb and Pratap, 2002) y (Kalami, 2010).

El algoritmo implementado parte de una poblacion inicial generada de forma aleatoria Po. Una vez se tiene la poblacion incial se genera la población descendiente Qo (esta se denota como Qt para futras generaiones). Posteriormente se calcula la función objetivo y se hace la clasificación de la pobalción por dominancia. Posteriormene se aplican los operadores de selección, cruce y mutacion. Para mantener la diversidad de la población se implementa el elitismo. Esto es, en todas las operaciones de selección, la densidad de la población en la vecindad de un individuo se utiliza como medida secundaria de adaptación. Cada vez que se comparan dos individuos, se emplea el comparador que permite saber cual está mejor adaptado. Se prefiere el individuo que pertenece al menor frente, y si ambos pertenecen al mismo frente se prefiere la solución con menos soluciones a su alrededor. En la Fig. 2 se ilustra el diagrama de flujo del NSGAIl implementado. 


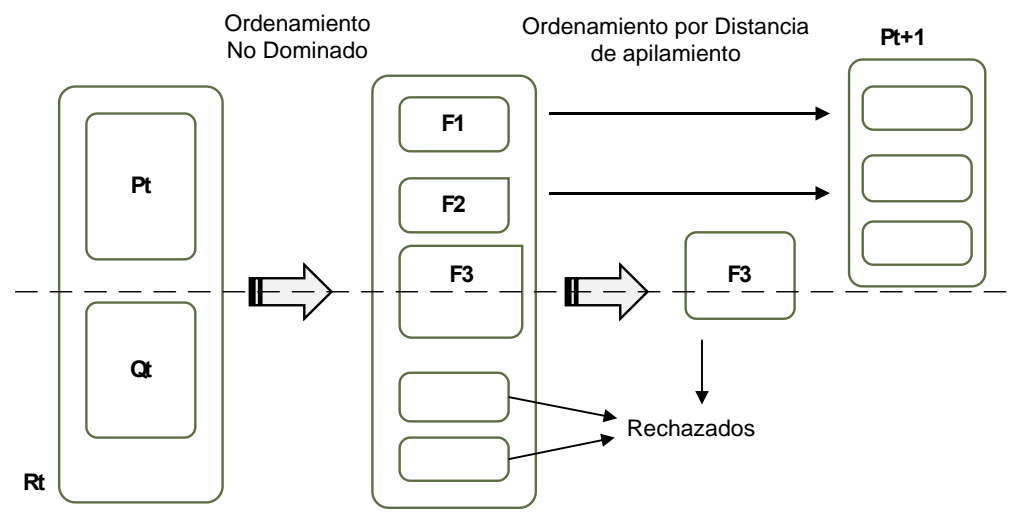

Fig. 1: Selección de individuos del NSGAII.

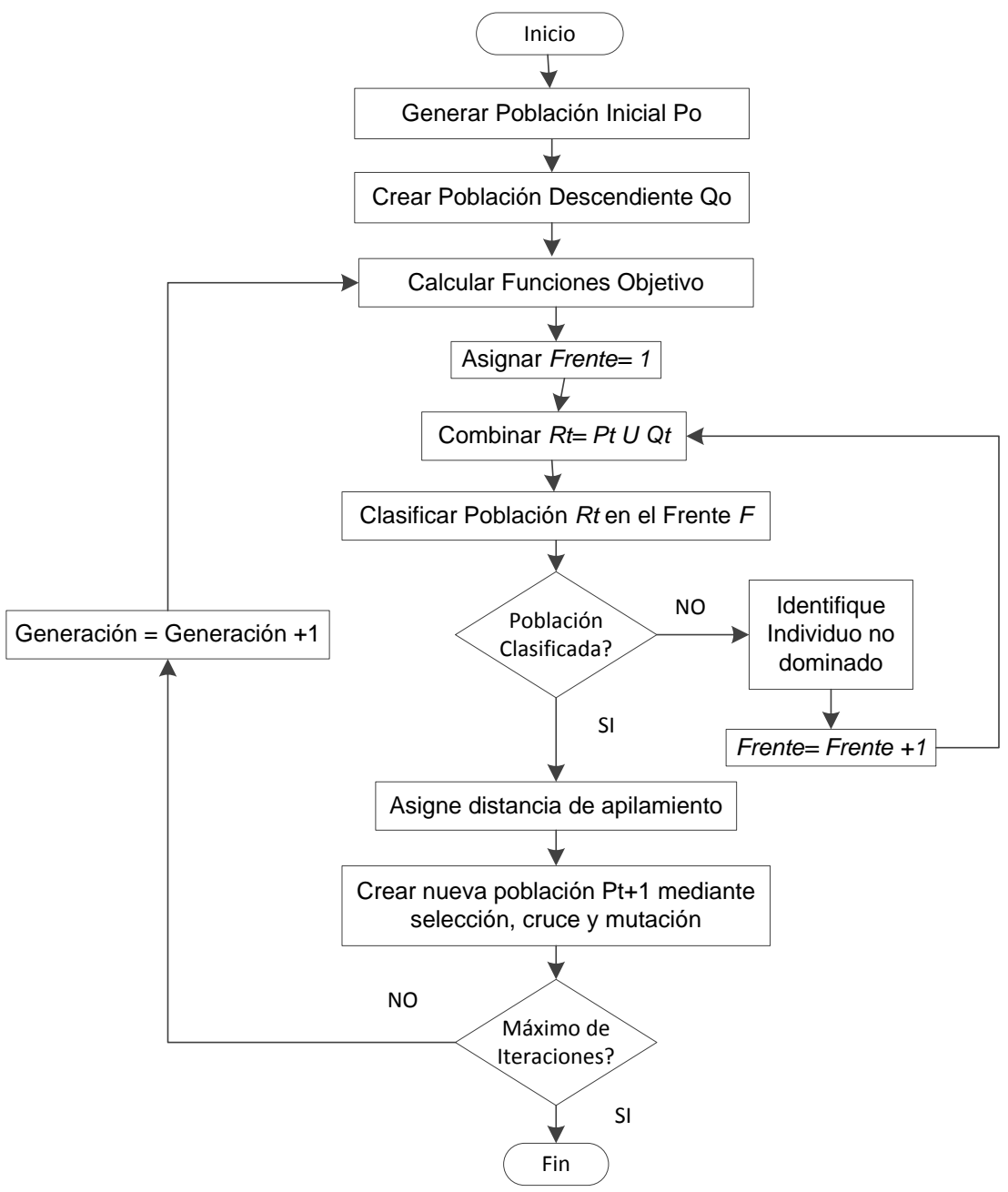

Fig. 2: Diagrama de flujo del NSGAll

\section{Algoritmo Memético Multiobjetivo (MOMA)}

Los Algoritmos Meméticos (AMs) son metaheurísticas basadas en población; esto quiere decir que mantienen un conjunto de soluciones candidatas para el problema considerado. Cada una de estas soluciones candidatas se denomina individuo o agente. Los AMs combinan ideas y conceptos de diferentes técnicas metaheurísticas como las técnicas evolutivas y las técnicas basadas en búsqueda local. En este sentido, un AM puede verse como un conjunto de agentes o individuos que realizan la exploración de un espacio de búsqueda interactuando a través de operadores como la recombinación y compitiendo por recursos computacionales a través de los mecanismos de selección y sustitución (Moscato y Cotta, 2003). 
Los AMs fueron inspirados por el principio de evolución darwiniano y por el concepto de meme introducido por Richad Dawkins (Dawkins, 1989). El término meme guarda similitud con "memoria". Un meme, en la teoría de difusión cultural, es la unidad teórica de información cultural transmisible de un individuo a otro, o de una mente a otra, o de una generación a la siguiente. La transmisión de los memes se da por las características propias de todo proceso evolutivo.

En este caso se implementó un MOMA (Multi-Objective Memetic Algorithm) siguiendo las recomendaciones de diseño y programación propuestas en (Knowles y Corne, 2005). La estructura del MOMA implementado incorpora los operadores de los algoritmos genéticos (selección, cruce, mutación), además de una Búsqueda Local (BL) como se indica en la Fig. 3. Primero se genera la poblacion incial Po de forma aleatoria, posteriormente se evalúa su función objetivo Co. Luego se hace la clasificación no dominada de la población para calcular el frente de Pareto, posteriormente se aplican los operadores comunes de los algoritmos genéticos para generar una nueva poblacion. El operador de cruce adiciona la característica de cooperación al pasar información de un individuo a otro. Por otro lado, la mutación hace que se introduzca información externa no contenida en el individuo. Finalmente, el operador de BL se puede aplicar en cualquier etapa de la fase reproductiva, ya sea después de la recombinación, mutación, a un subconjunto de soluciones o al final del ciclo reproductivo. En este caso la BL se aplica al final del ciclo reproductivo. Una descripción más detallada de los Algoritmos Meméticos y su aplicación a problemas mono y multiobjetivo puede ser consultada en Knowles y Corne (2005) y Geng y Wenxing (2014).

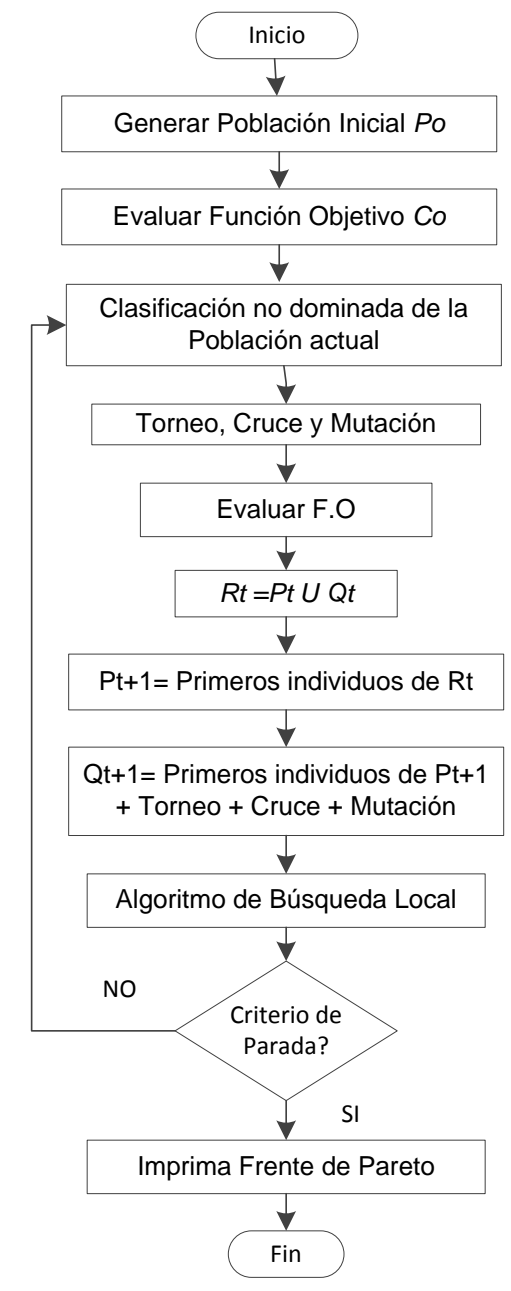

Fig. 3: Diagrama de flujo del MOMA

\section{PRUEBAS Y RESULTADOS}

Los algoritmos propuestos fueron probados en dos circuitos rurales de 100 y 200 nodos pertenecientes al sistema de distribución de energía eléctrica del departamento de Antioquia en Colombia. Dichos circuitos suministran energía a clientes rurales ubicados en zonas con características geográficas y contexto operacional similares. En la Tabla 2 se muestran los datos de los circuitos que conforman los casos de prueba. 
Tabla 2. Características de los casos de prueba de los algoritmos

\begin{tabular}{|l|c|c|}
\hline Tamaño del caso [nodos] & 100 & 200 \\
\hline Longitud de red [km] & 1268 & 2220 \\
\hline Número de Clientes & 13245 & 26839 \\
\hline Costo de Mantenimiento [Millones COP] & 1858 & 3253 \\
\hline Máxima mejora SAIFI & 5,886 & 5,681 \\
\hline
\end{tabular}

Para hacer pruebas y calibrar los algoritmos inicialmente se tomó el caso de prueba más exigente en tamaño (200 nodos), con el objetivo de determinar la cantidad de individuos y el número de generaciones, con respecto al tiempo, para obtener el mejor desempeño del algoritmo. Inicialmente se realizó el experimento con el MOMA y se reprodujo el resultado en el NSGA II. En Fig. 4 y Fig. 5 se ilustran los resultados obtenidos. Los algoritmos fueron programados y ejecutados en Matlab®, versión R2010a, de 64 bit (win 64), y fueron ejecutados en un computador con un procesador Intel @ Core ${ }^{\mathrm{TM}}$ i5-3317U@1.7 Ghz, RAM de 4GB, sistema operativo de 64 bits procesador X64.

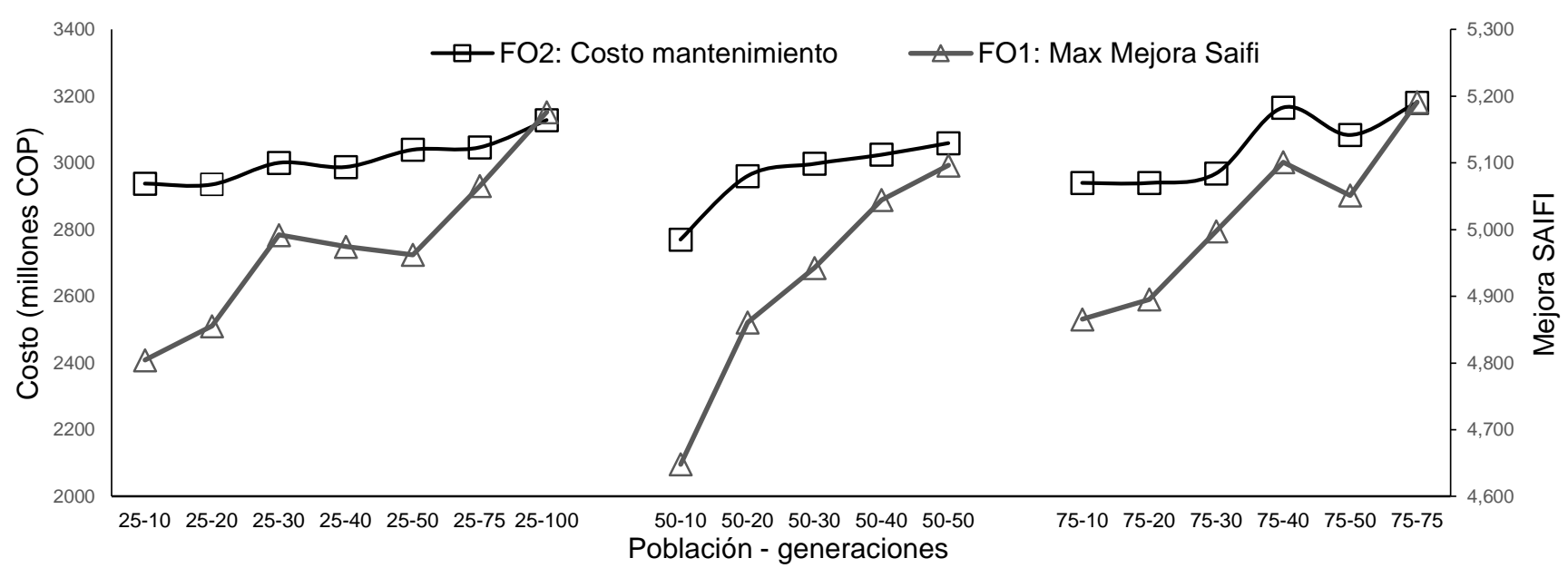

Fig. 4: Funciones objetivo vs. número de generaciones (sistema de 200 nodos)

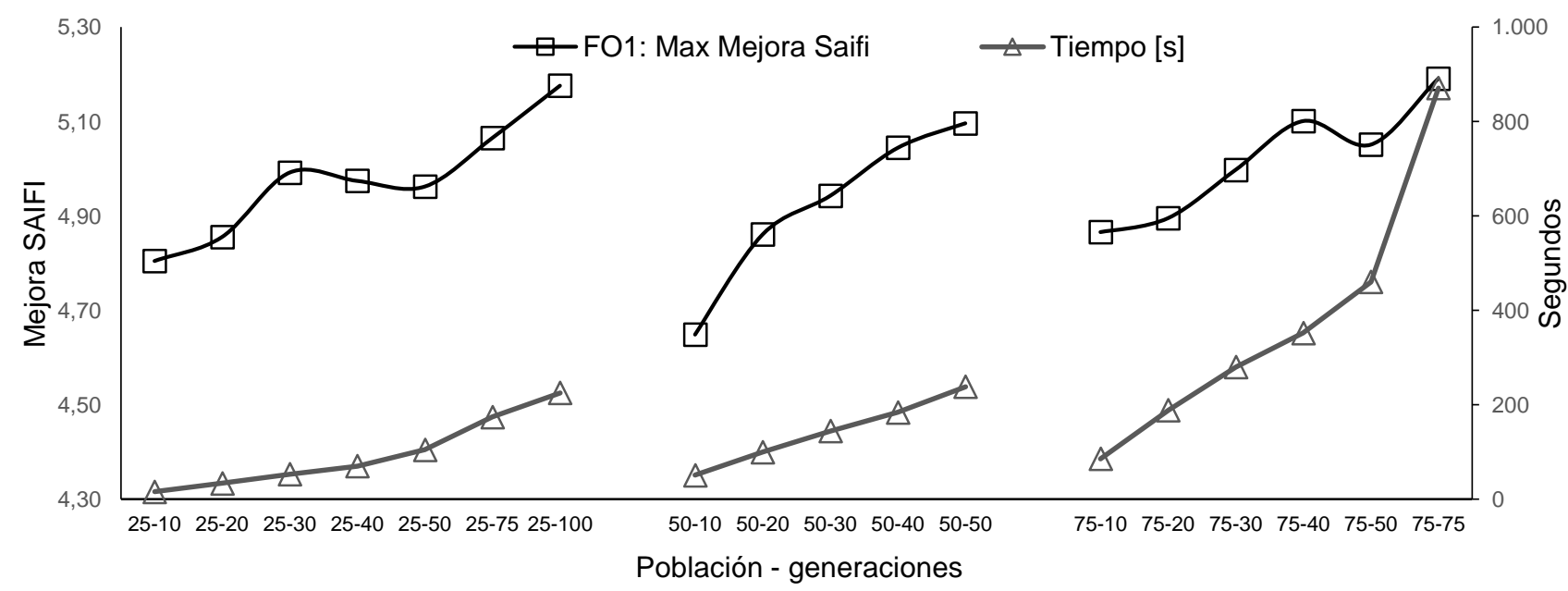

Fig. 5: Mejora SAIFI vs. tiempo de ejecución (sistema de 200 nodos)

Se encontró que a mayor número de generaciones se obtiene una mejor función objetivo 1, con una desviación del $9 \%$ con respecto a la máxima mejora del SAIFI en un tiempo promedio de 225 segundos. La búsqueda local (BL) se hace para el $20 \%$ de los individuos a partir de un punto aleatorio seleccionado. Posteriormente se realizaron pruebas para el sistema de 100 nodos. En la Tabla 3 se resume, como referencia, el ajuste fino para ambos sistemas de prueba. Esto se hace con el fin de ajustar los parámetros del algoritmo en términos de poblacion y número de iteraciones. De esta forma se determina la cantidad de individuos (población) y el número de generaciones (iteraciones) versus el tiempo con el propósito de 
obtener el mejor desempeño del algoritmo. Es importante notar que el caso de prueba de 100 nodos, presenta el mejor desempeño con una población de 50 individuos y 30 iteraciones, con un tiempo de ejecución de 87 segundos. Las combinaciones 50-40 y 50-50 no mejoran la función objetivo y se alcanzan tiempos hasta de 150 segundos. Entonces el valor de 50-30 se considera como el ajuste de referencia para el problema de 100 nodos. Como regla general se considera que para problemas de mayor tamaño se aconseja tener poblaciones de menos cantidad de individuos y más iteraciones.

Tabla 3. Ajuste fino de Población - Número de iteraciones para los sistemas de prueba.

\begin{tabular}{|c|c|c|c|c|c|c|}
\hline \multirow{2}{*}{$\begin{array}{l}\text { Número } \\
\text { nodos }\end{array}$} & \multirow{2}{*}{$\begin{array}{l}\text { Población - } \\
\text { iteraciones }\end{array}$} & \multirow{2}{*}{$\begin{array}{c}\text { FO1 Mejora } \\
\text { SAIFI }\end{array}$} & \multirow{2}{*}{$\begin{array}{c}\text { FO2 Costo } \\
\text { Mantenimiento }\end{array}$} & \multicolumn{2}{|c|}{ Porcentaje desviación } & \multirow[t]{2}{*}{ Tiempo [s] } \\
\hline & & & & FO1 & FO2 & \\
\hline 100 & $50-30$ & 5,750 & 1494 & $2 \%$ & $9 \%$ & 87 \\
\hline 200 & $25-100$ & 5,176 & 3129 & $9 \%$ & $4 \%$ & 225 \\
\hline
\end{tabular}

En Fig. 6, Fig. 7, Fig. 8 y Fig. 9 se ilustra el desempeño de ambos algoritmos en cuanto al tiempo de ejecución y a la desviación de la máxima mejora del SAIFI para los casos de prueba estudiados. Cada algoritmo se corrió cinco veces por cada combinación de población y número de iteraciones. Posteriormente, se calcularon los promedios por corrida. En total, cada algoritmo se ejecutó 280 veces. Como se observa en Fig. 6 el MOMA siempre supera al NSGA II en el porcentaje de desviación con respecto a la máxima mejora del SAIFI. Sin embargo, en cuanto al tiempo de ejecución el NSGA II mostró ser más veloz (ver Fig. 7). Se resalta que el MOMA funciona muy bien con problemas de gran tamaño; por ejemplo, para el problema de 200 nodos este algoritmo tiene un porcentaje de desviación del $9 \%$, mientras que para el NSGA II es del $15 \%$.

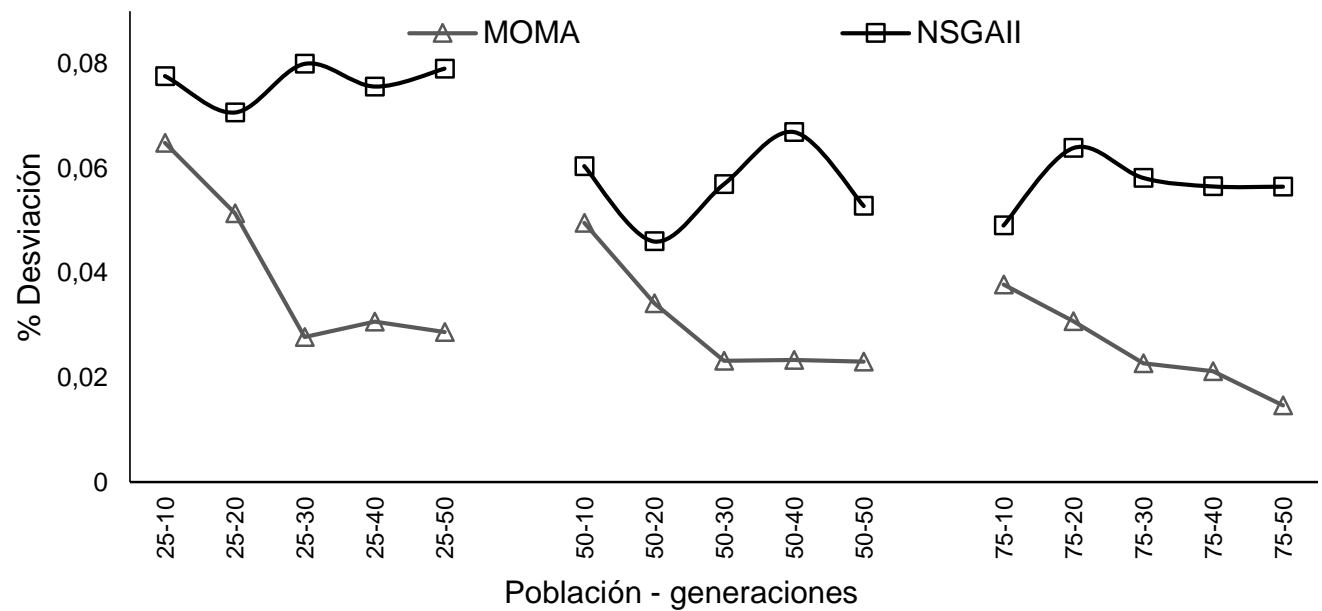

Fig 6. Desvaicon con respecto a la máxima mejora del SAIFI. Sistema de 100 nodos

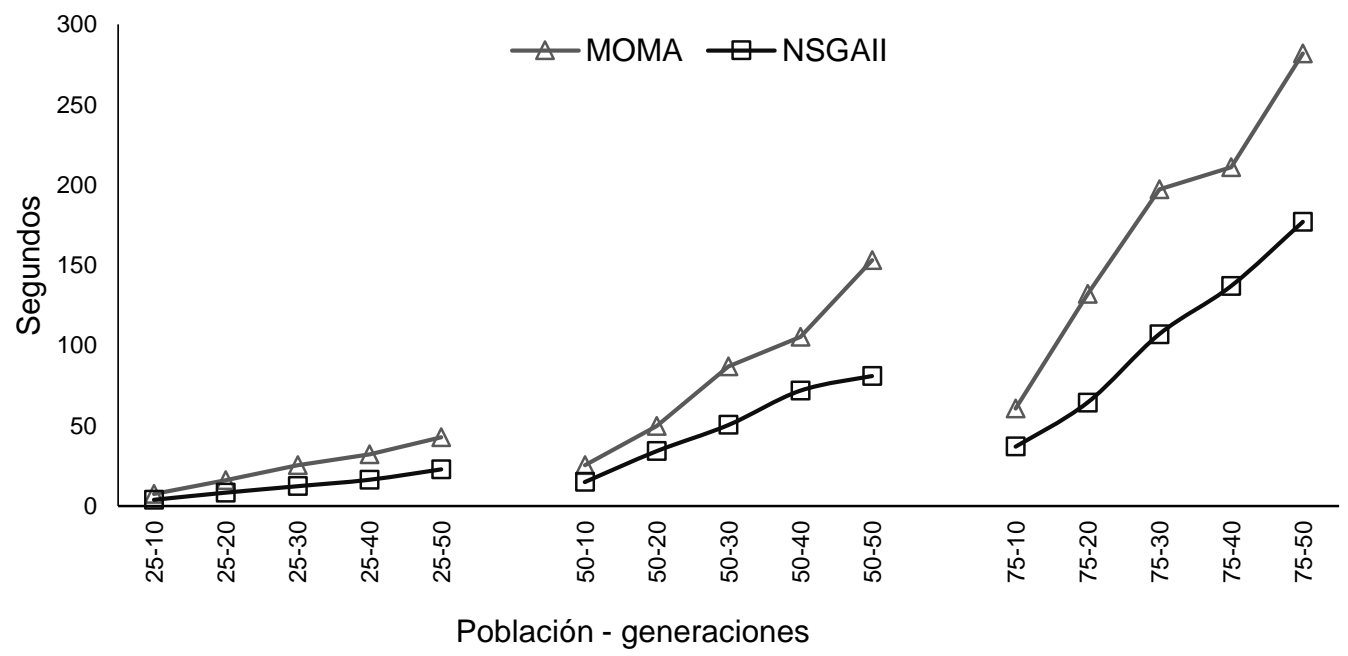

Fig. 7: Tiempos de ejecución para el sistema de 100 nodos 


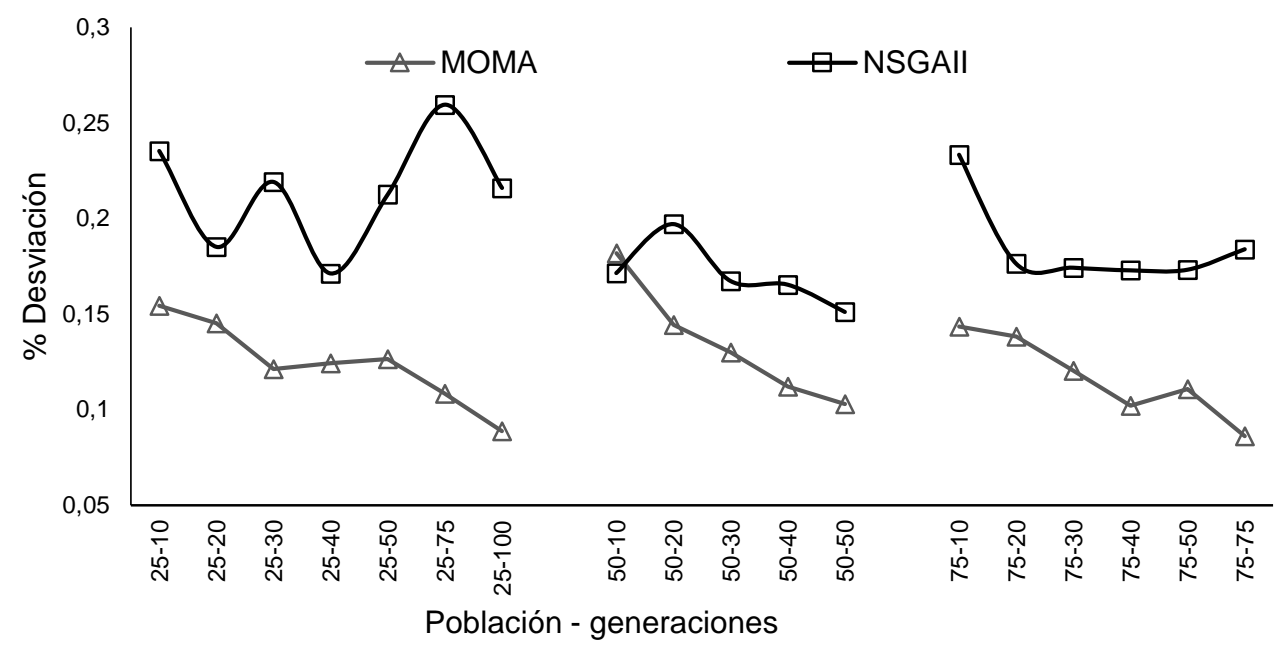

Fig. 8: Desvaicon con respecto a la máxima mejora del SAIFI. Sistema de 200 nodos

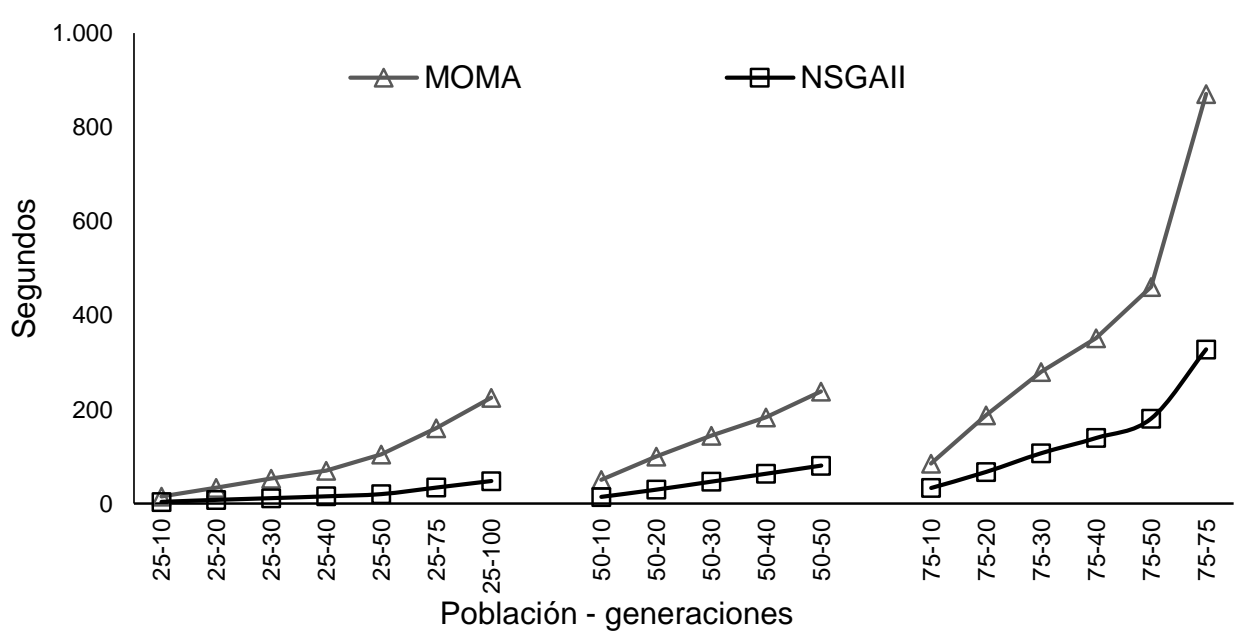

Fig. 9: Tiempos de ejecución para el sistema de 200 nodos

En la revisión de los tiempos empleados por el MOMA para resolver el problema de 200 nodos se encuentra que como era de esperarse, el mayor tiempo lo emplea el módulo de ordenamiento por no dominancia (compara las posibles soluciones una a una) y luego el operador de Búsqueda Local (se ejecuta en $20 \%$ de los individuos). Para el problema específico que se pretende resolver se considera que el tiempo no es crítico, mientras que el porcentaje de error al óptimo global si puede representar un considerable ahorro en costos para las empresas de distribución.

Con el fin de determinar la estabilidad y la reproducibilidad de los resultados del MOMA, se determinó el coeficiente de variación para 280 corridas del algoritmo. Se encontró, un coeficiente de variación de 3,76\% para el problema más grande (200 nodos). Este valor se considera de muy buena calidad y comprueba el potencial de uso del MOMA aplicado a problemas de gran tamaño. En la Tabla 4 se pueden ver los valores para los otros casos de prueba del algoritmo. En Fig. 10 y Fig. 11 se presentan los frentes óptimos de Pareto obtenidos para los sistemas de 100 y 200 nodos, con ambas metodologías, respectivamente. Una de las ventajas de la metodología propuesta es que es fácilmente aplicable a sistemas de tamaño real como los presentados en este artículo. Adicionalmente, el operador de red puede decidir sobre un conjunto de soluciones que representan un compromiso entre el mejoramiento del indicador de calidad y el costo asociado con el mismo.

Tabla 4. Coeficiente de variación del MOMA, aplicado a los casos de prueba.

\begin{tabular}{|c|c|c|}
\hline Número de nodos & 100 & 200 \\
\hline Desviación Estándar & 0,0225 & 0,0374 \\
\hline Promedio desviación & 0,9985 & 0,9931 \\
\hline Coeficiente de variación & $2,25 \%$ & $3,76 \%$ \\
\hline
\end{tabular}




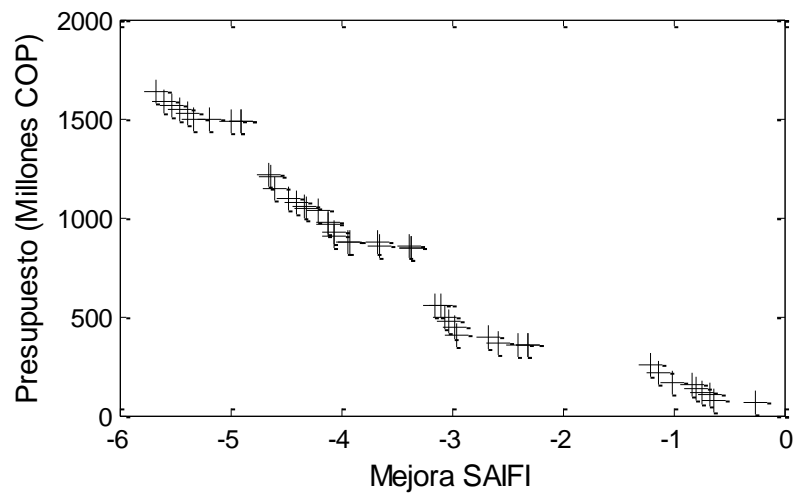

a) MOMA

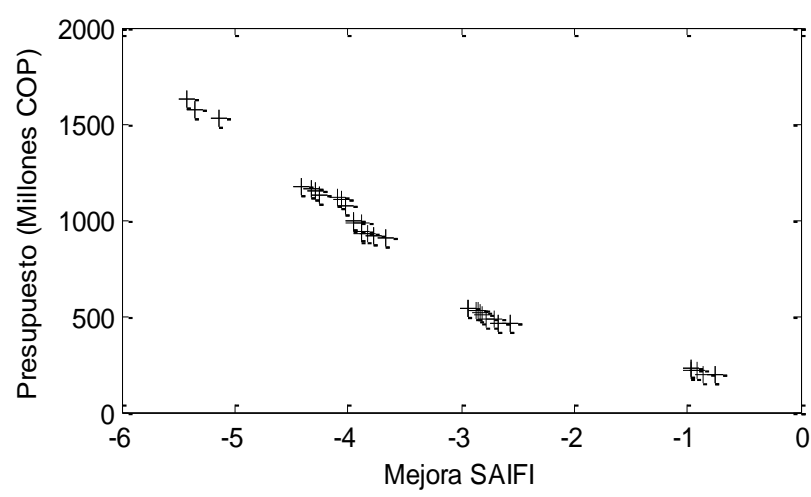

b) NSGAII

Fig. 10: Frentes óptimos de Pareto para el sistema de 100 nodos

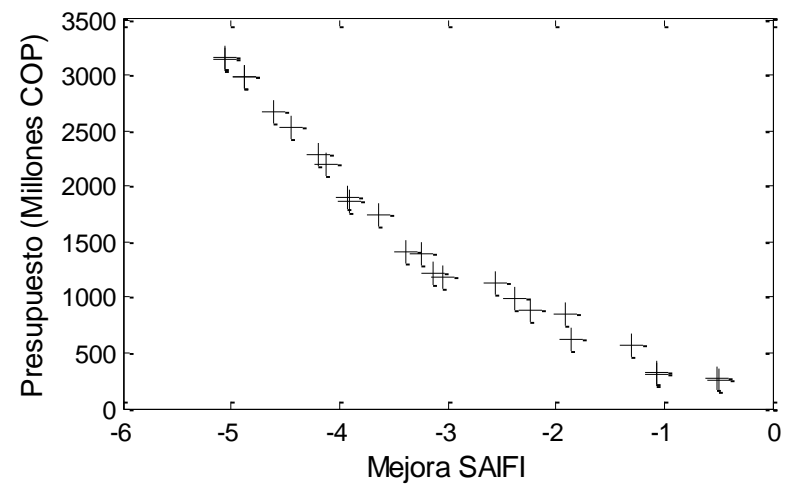

a) MOMA

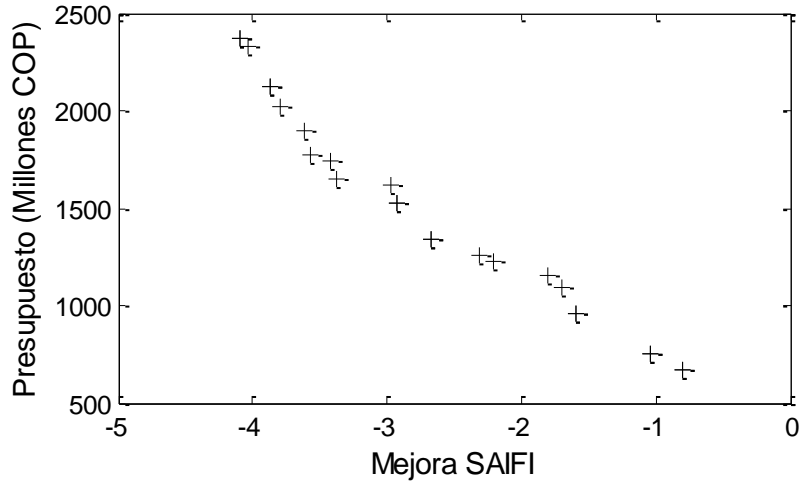

b) NSGAII

Fig. 11: Frentes óptimos de Pareto para el sistema de 200 nodos

Se puede observar que para el caso de 100 nodos la calidad de las soluciones obtenidas con ambos algoritmos resultan ser muy similares. En este caso, la máxima mejora en el SAIFI se puede obtener con un costo de 1600 millones de pesos colombianos (COP). Por otro lado, se pueden obtener mejoras intermedias al indicador a menor costo como se indica en Fig. 10. En Fig. 11 se evidencia que el MOMA pudo obtener un frente de Pareto un poco más extenso encontrando posibles soluciones con mejores indicadores SAIFI. En ambos casos el operador de la red de distribución cuenta con un set de posibles soluciones y deberá seleccionar la más apropiada según su relación costo-beneficio.

\section{CONCLUSIONES}

En este artículo se compararon dos metodologías para asignación óptima de presupuesto en sistemas de distribución. Las metodologías propuestas (NSGAll y MOMA) buscan maximizar la mejora en el índice de fallas (SAIFI) y minimizar el costo de mantenimiento. Se realizaron pruebas con sistemas reales de $100 \mathrm{y}$ 200 nodos. Los algoritmos implementados mostraron ser robustos y efectivos para solucionar el problema bajo estudio. En cuanto a velocidad de cómputo el NSGAll resultó ser más rápido que el MOMA, pues este último incorpora una búsqueda local que requiere un tiempo de computo considerable. Para el sistema de 100 nodos ambos algoritmos mostraron desempeño similar en cuanto a la calidad de las soluciones encontradas. Sin embargo, para el caso de 200 nodos el MOMA se mostró superior al NSGAll en este aspecto. Se puede concluir que para sistemas de gran tamaño el MOMA puede encontrar mejores soluciones que el NSGAll, aunque con un tiempo de cálculo mayor. Los algoritmos implementados permiten identificar el nivel de presupuesto que debe ser destinado para alcanzar una mejora especificada en el SAIFI. La principal ventaja de la metodología presentada en este artículo consiste en obtener un conjunto de soluciones que representan un compromiso entre los dos objetivos bajo estudio. Esto permite al planeador de la red de distribución comparar entre diferentes alternativas y estimar qué niveles del indicador SAIFI puede alcanzar con determinado presupuesto. 


\section{REFERENCIAS}

Abiri-Jahromi A., M. Fotuhi-Firuzabad, and E. Abbasi, An Efficient Mixed-Integer Linear Formulation for Long-Term Overhead Lines Maintenance Scheduling in Power Distribution Systems, IEEE Trans. Reliab., 24(4): 2043-2053, (2009)

Arias, A., R.A. Hincapie, M. Granada and R.A. Gallego, Optimal scheduling of vegetation maintenance underneath overhead power distribution lines, IEEE Transmission and Distribution Conference and Exposition Latin America, Montevideo, pp. 1-6, (2012)

Arya, R., and C. Choube, Differential evolution based technique for reliability design of meshed electrical distribution systems. International Journal of Electrical Power and Energy Systems, 48(2): pp.10-20, (2013)

Bahri, O., N. Ben Amor and T. El-Ghazali, Optimization algorithms for multi-objective problems with fuzzy data, IEEE Symposium on Computational Intelligence in Multi-Criteria Decision-Making (MCDM), Orlando, Florida, pp. 194-201, (2014)

Certa, A., G. Galante, T. Lupo and G. Passannanti, Determination of Pareto frontier in multi-objective maintenance optimization, Reliability Engineering and System Safety, 96(7): 861-867, (2011)

Deb, K., and A. Pratap, A fast and elitist multiobjective genetic algorithm: NSGA-II, IEEE Transactions on Evolutionary Computing, 6(2): pp. 182-197, (2002)

Dawkins, R. The Selfish Gen, Oxford University Press, Second Edition, (1989)

García, C., E. García and F. Villada, Algoritmo Evolutivo Eficiente Aplicado a la Planeación de la Expansión de Sistemas de Distribución, Revista Información Tecnológica 23(4): pp. 3-10, (2012)

Geng L. and Z. Wenxing, An efficient Memetic Algorithm for the Max-Bisection Problem, IEEE Transactions on Power Computing, 63(6): pp. 1365-1376, (2014)

Ghoreishi, S.N., J.C. Sorensen, and B.N. Jorgensen, Comparative study of evolutionary multi-objective optimization algorithms for a non-linear Greenhouse climate control problem, IEEE Congress on Evolutionary Computation, Sendai, Japan, pp. 1909-1917, (2015)

Hajebrahimi, A., M. Rashidinejad, and A. Abdollahi, A fuzzy Analysis to connect the large-scale distant wind farm to grid in probabilistic multi objective transmission expansion planning, 22th Iranian Conference on Electrical Engineering, Tehran, Iran, pp. 589-595, (2014)

Kalami, M., A version of the NSGA-II in MatLab, 2010. (Accessed: 10-Nov-2014) [Online]. Available: http://delta.cs.cinvestav.mx/ ccoello/EMOO/EMOOsoftware.html. (2010)

Knowles J. and D. Corne, Memetic algorithms for multiobjective optimization: issues, methods and prospects, Studies in Fuzziness and Soft Computing, 166(1): pp. 313-352, (2005)

Mazidi, M.R., M. Aghazadeh, Y.A. Teshnizi, and E. Mohagheghi, Optimal placement of switching devices in distribution networks using multi-objective genetic algorithm NSGAll, 21th Iranian Conference on Electrical Engineering, Tehran, Iran, pp. 1-6, (2013)

Moscato, P., y C. Cotta, Una Introducción a los Algoritmos Meméticos, Revista Iberoamericana de Inteligencia Artificial,19(19): pp.131-148, (2003)

Narváez, P.A., J.M. López-Lezama, and E. Velilla, Ubicación de Generación Distribuida para Minimización de Pérdidas Usando un Algoritmo Genético Híbrido. Revista Información Tecnológica, 26(3): po.123-132, (2015)

Sun, J., J. Garibaldi, N. Krasnogor, and Q. Zhang, An Intelligent Multi-Restart Memetic Algorithm for Box Constrained Global Optimisation, Evolutionary computation, 21(1): pp.107-147, (2014)

Teera-archariyakul, N., K. Chulakhum, D. Rerkpreedapong, and P. Raphisak, Optimal allocation of maintenance budgets for reliability Target setting, Asia and Pasific Power and Energy Engineering Conference, Wuhan, China, pp. 1-6, (2010)

Yongmei, L.I., M. Xiaoli, and S. Wanxing, Research and application of maintenance schedule optimization based on intelligent algorithm, International Conference on Advanced Power System Automation and Protection Research, Beijing pp. 1-6, (2011)

Xianchao, H., Z. Lizi, S. Jun, and Z. Jingwei, Distribution maintenance time scheduling using a genetic algorithm. IEEE Power Systems Technology Conference, India, pp. 1-6, (2008) 
